\title{
Improved Method for the Identification of the Fluoride-Resistant Plasmacholinesterase Genotypes ${ }^{1}$
}

\author{
E. K. Zsigmond, ${ }^{2,3}$ Kathleen B. Flynn, ${ }^{2}$ and S. P. Kothary
}

Received 20 June-Final 2 Nov. 1979

This investigation was prompted by the findings that (1) dibucaine-resistant homozygotes and heterozygotes for plasmacholinesterase also exhibit resistance to fluoride inhibition, (2) the differentiation of dibucaine-resistant from the fluoride-resistant genotypes is ambiguous with the method of Harris and Whittaker, (3) the plasmacholinesterase inhibition by Na fluoride $(F N)$ is markedly influenced by the temperature. Therefore, we modified their method by increasing (1) the temperature of the reaction from $25 \mathrm{C}$ to $37 \mathrm{C}$ and (2) the concentration of $\mathrm{Na}$ fluoride from $5.0 \times 10^{-5} \mathrm{M}$ to $2.5 \times$ $10^{-4} \mathrm{M}$. With this method, genetically normal individuals have a mean $F N \pm$ $S D=77.0 \pm 3.22$ while atypical dibucaine-resistant homozygotes have a mean $F N \pm S D=43.0 \pm 10.0$ and atypical dibucaine-resistant heterozygotes $67.0 \pm 5.37$. Since a linear correlation was observed between DN and FN by our new method, a fluoride number $2 S D$ lower than the predicted $F N$ from the $D N$ can distinctly identify the fluoride-resistant plasmacholinesterase genotype $E_{1}^{f}$.

KEY WORDS: FN determination; plasmacholinesterase; $E_{1}^{f}$ genotype; enzyme assay; modified FN.

\footnotetext{
${ }^{1}$ A preliminary report was presented at the annual meeting of the American College of Clinical Pharmacologists, April 29-May 1, 1971.

${ }^{2}$ Department of Anesthesiology and Anesthesia Research Laboratories, University of Michigan Medical Center, Ann Arbor, Michigan 48109.

${ }^{3}$ Present address: Department of Anesthesiology, University of Illinois Medical Center, Chicago, Illinois 60612 .
} 


\section{INTRODUCTION}

Harris and Whittaker (1961) claimed that a new phenotype of human serum cholinesterase was recognized by sodium fluoride inhibition. Since this original report, the method of Harris and Whittaker has been accepted as a standard method for the determination of fluoride-resistant plasmacholinesterase (Whittaker, 1967, 1968; Thompson and Whittaker, 1966; Whittaker and Vickers, 1970). These authors utilized $5.0 \times 10^{-5} \mathrm{M}$ concentration of $\mathrm{Na}$ fluoride at 25C. Under these conditions of essay, a normal person has $\mathrm{FN}<$ 64 while a fluoride-resistant homozygote $E_{1}^{f} E_{1}^{f}$ ) has $\mathrm{FN}<34$.

After the adaptation of the method for FN determination according to Harris and Whittaker, we early recognized that the narrow percentage ranges of inhibition in the various genotypes with the use of $5.0 \times 10^{-5} \mathrm{M} \mathrm{Na}$ fluoride concentration did not allow a clear differentiation of the genotypes. Therefore, a great overlap existed between the various genotypes based on the concomitant determination of DN by the method of Kalow and FN by the method of Harris and Whittaker. Subsequently we recognized that individuals with dibucaine resistance also exhibited fluoride resistance and vice versa at both 25 and $37 \mathrm{C}$ utilized by Foldes et al. (1963) for the determination of DN. Although the increase in temperature from 25 to $37 \mathrm{C}$ caused only predictable and minor changes in DN, it markedly influenced the FN. In order to improve the selectivity and accuracy of FN determination, our study was undertaken.

\section{MATERIALS AND METHODS}

\section{Source of Enzymes}

Freshly obtained venous heparinized blood samples were separated by centrifugation at $2600 \mathrm{rpm}$ into red cells and plasma. The supernatant plasma was immediately separated from the buffy coat and stored at $-20 \mathrm{C}$ or immediately utilized. The hydrolysis rate of benzoylcholine chloride commercially obtained from Calbiochem (Pasadena, California) was determined by Kalow's ultraviolet spectrophotometric method at 25,31 , and $37 \mathrm{C}$ as indicated in the figures. The influence of dibucaine hydrochloride, sodium fluoride, and RO2-0683 [2-hydroxy-5-phenylbenzyl trimethylammonium (bromide) dimethylcarbamate] on the hydrolysis of benzoylcholine chloride was determined, and $p \mathrm{I}$-inhibition curves were plotted as shown in Results. Each point represents the mean of three determinations checking within 5\%. Based on the $p \mathrm{I}-$ inhibition curves, a concentration of $\mathrm{Na}$ fluoride was selected which gives $75 \%$ inhibition of benzoylcholine hydrolysis rate. In addition, 
RO2-0683 was utilized to confirm the dibucaine resistant genotypes as reported by Foldes et al. (1963). Three plasma samples of suspected or claimed fluoride-resistant plasma cholinesterase genotypes were also studied and the $p \mathrm{I}$-inhibition curves of the three inhibitors determined at $37 \mathrm{C}$.

\section{RESULTS}

The $p \mathrm{I}$ inhibition curves of $\mathrm{Na}$ fluoride at $37 \mathrm{C}$ and $25 \mathrm{C}$ in the plasma of normal $E_{1}^{u} E_{1}^{u}$ and atypical dibucaine-resistant homozygotes $E_{1}^{a} E_{1}^{a}$ are shown in Fig. 1. It is evident that increasing the reaction temperature from 25 to $37 \mathrm{C}$ results in a markedly reduced $\mathrm{FN}$. It can also be observed that there is a marked difference in the $I_{50}$ between the $E_{1}^{u} E_{1}^{u}$ and $E_{1}^{a} E_{1}^{a}$. It is evident from the curve that the use of $5.0 \times 10^{-5} \mathrm{M}$ concentration at either 25 or $37 \mathrm{C}$ may result in a very large experimental error because this concentration falls on the mid portion of the $p \mathrm{I}$-inhibition curve where the slope is the greatest.

Figures 2 and 3 summarize the results obtained with dibucaine and RO2-0683. As can be observed in Fig. 2, the influence of temperature on the pI-inhibition curve of dibucaine is small. Consequently, changing the temperature from 25 to $37 \mathrm{C}$ results in only a few units of change in DN. It should also be noted that $1 \times 10^{-5} \mathrm{M}$ dibucaine concentration falls on the flattening portion of the $p \mathrm{I}$-inhibition curve; therefore, errors in dibucaine concentration will lead to a little experimental error in DN determination. Similarly the $p \mathrm{I}$-inhibition curve of $\mathrm{RO} 2-0683$ was little affected by temperature change, as Fig. 3 illustrates.

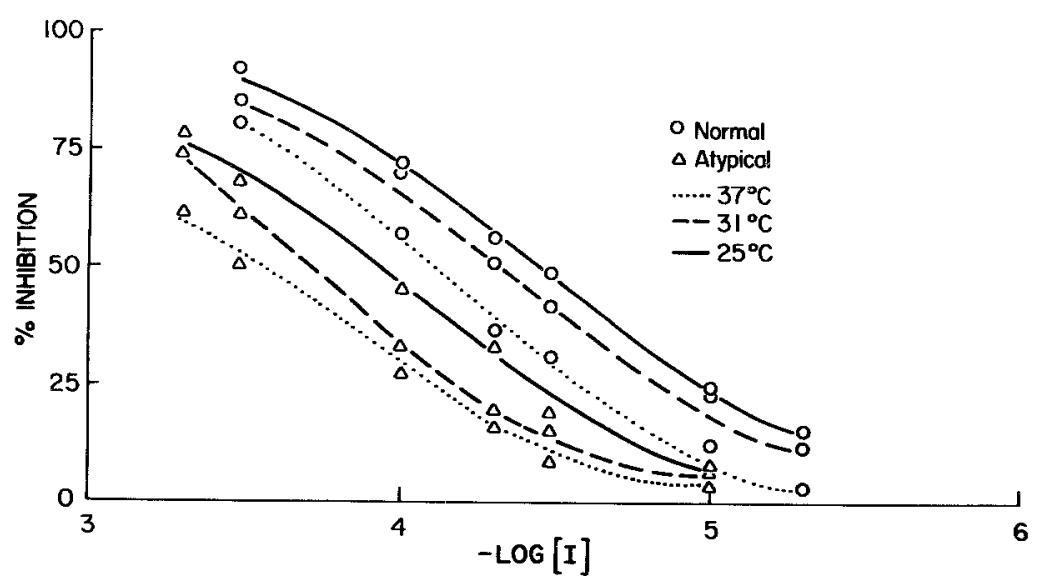

Fig. 1. Effect of temperature on the in vitro inhibition of normal and atypical homozygous plasmacholinesterase by sodium fluoride. 


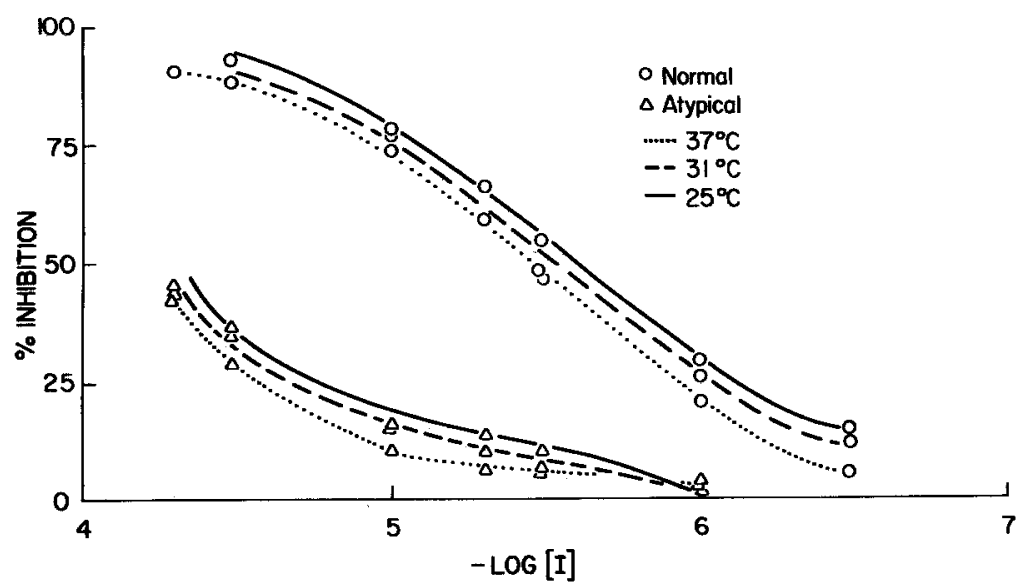

Fig. 2. Effect of temperature on the in vitro inhibition of normal and atypical homozygous plasmacholinesterase by dibucaine hydrochloride.

A distinct differentiation of the various genotypes can be achieved by the utilization of the $p \mathrm{I}$-inhibition curves of dibucaine, sodium fluoride, and RO2-0683 in combination. In this way, three claimed $E_{1}^{u} E_{1}^{f}$ variants were unquestionably identified as $E_{1}^{a} E_{1}^{a}$ or $E_{1}^{u} E_{1}^{a}$. Figure 4 shows such an example of $E_{1}^{a} E_{1}^{a}$ who was erroneously classified as a fluoride-resistant heterozygote $E_{1}^{a} E_{1}^{f}$ (compare Fig. 2 to Fig. 4). Figure 5 shows the effect of sodium fluoride on the cholinesterase of the same $E_{1}^{a} E_{1}^{a}$ (compare Fig. 5 to Fig. 1).

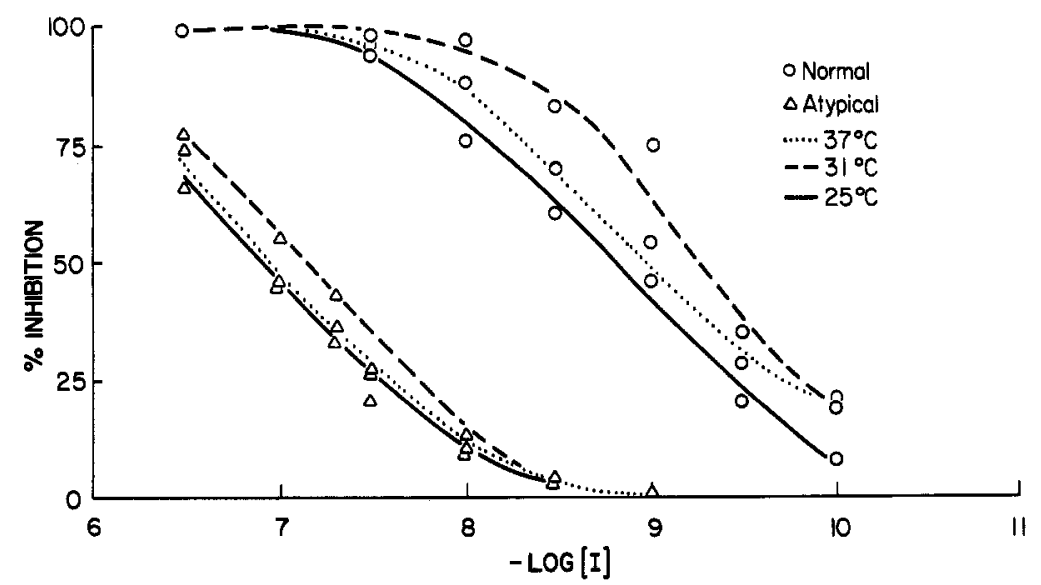

Fig. 3. Effect of temperature on the in vitro inhibition of normal and atypical homozygous plasmacholinesterase by RO2-0683. 


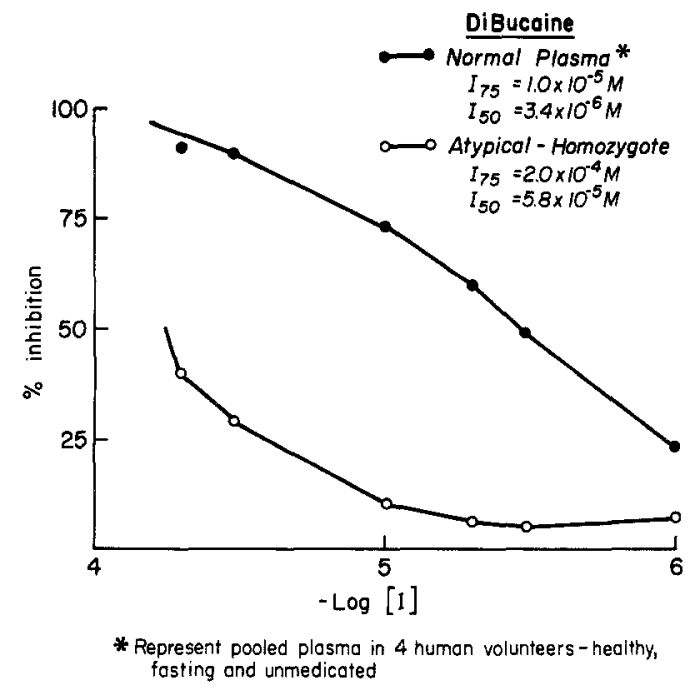

Fig. 4. Identification of the genotype of plasma cholinesterase by the $p \mathrm{I}$-inhibition curve for dibucaine in a questionable subject.

\section{DISCUSSION}

Since the discovery of fluoride-resistant genotype by Harris and Whittaker (1961), it has become increasingly evident that the differentiation of fluoride resistant from the dibucaine-resistant genotypes by this method was inaccu-

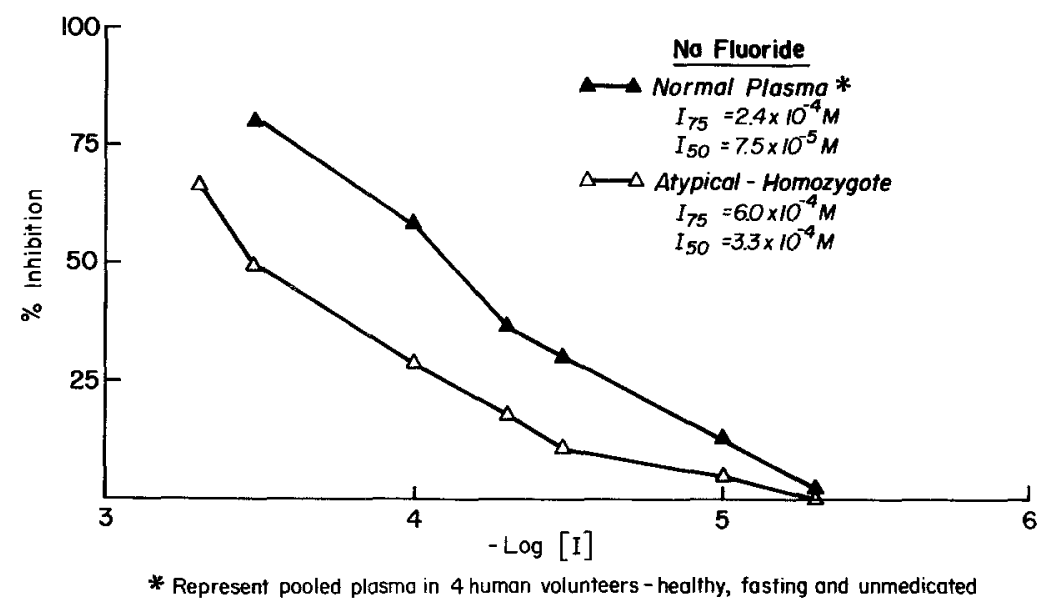

Fig. 5. Identification of the genotype of plasmacholinesterase by the $p \mathrm{I}$-inhibition curve for sodium fluoride in a questionable subject. 


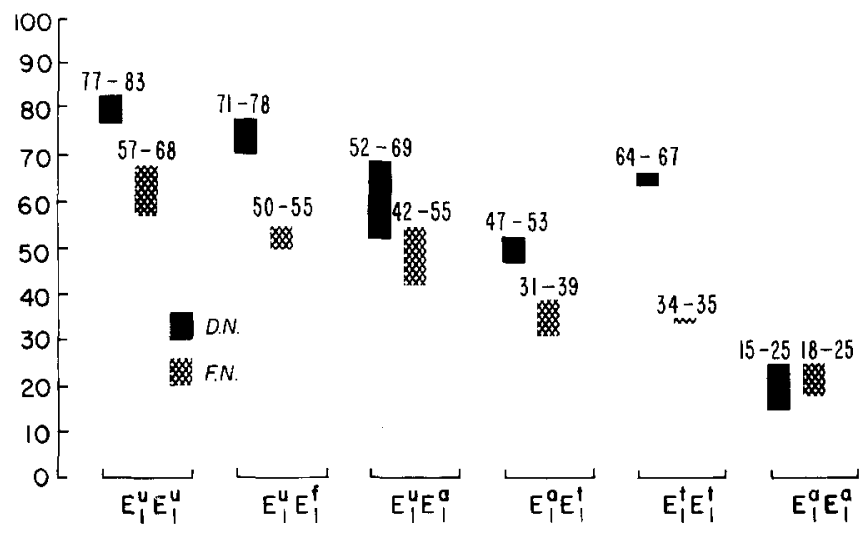

Fig. 6. DN and FN reported in 390 apneic patients with various atypical genotypes of plasmacholinesterase. Data plotted from Whittaker and Vickers (1970).

rate as is illustrated in Fig. 6. This figure contains all data points reported by Whittaker and Vickers (1970) from 390 apneic patients collected from the world literature. When these results were replotted, it became evident that based only on the DN and FN by the Harris and Whittaker method, genotypes $E_{1}^{u} E_{1}^{a}$ and $E_{1}^{a} E_{1}^{f}$ can be erroneously identified because of the overlap DN and FN values in these two groups. Furthermore, $E_{1}^{f} E_{1}^{f}$ cannot be distinguished from $E_{1}^{a} E_{1}^{f}$, since both FN and DN are intermediary, and the expected FNs fall between the values for normal homozygotes $\left(E_{1}^{u} E_{1}^{u}\right)$ and dibucaine-resistant homozygotes $\left(E_{1}^{a} E_{1}^{a}\right)$, as seen in Fig. 6.

That dibucaine-resistant homozygotes and heterozygotes also have an abnormally low FN was recently well established by Kothary et al. (1979). By the aid of a correlation equation between $\mathrm{DN}$ and $\mathrm{FN}, \mathrm{FN}=0.59 \mathrm{DN}+$ 32 , one can predict the $\mathrm{FN}$ in a dibucaine-resistant $E_{1}^{a}$ variant. No such fluoride-resistant variant $\left(E_{1}^{f}\right)$ was observed in 144 probands and their relatives referred to our laboratory, since none deviated from this equation. In order to clearly identify the genotypes containing an $E_{1}^{f}$ component, one is forced to resort to the plotting of the $p \mathrm{I}$-inhibition curve of dibucaine, $\mathrm{Na}$ fluoride, and RO2-0683 in the plasma samples of each individual claimed to be a fluoride-resistant phenotype. In three questionable $E_{1}^{f}$ variants, the $p \mathrm{I}$-inhibition curves using dubucaine, $\mathrm{Na}$ fluoride, and $\mathrm{RO} 2-0683$ clearly established that they were atypical dibucaine-resistant genotypes; one example is shown in Fig. 4.

Although it was claimed that phenotypes with an $E_{1}^{f}$ variant have prolonged apnea following succinylcholine by Harris and Whittaker (1961) and Whittaker and Vickers (1970), one should confirm the existence of such an $E_{1}^{f}$ variant by the $p \mathrm{I}$-inhibition curve, and/or simply by the determination of FN by our improved method, before one accepts such a cause-effect 
relationship. Erroneous identification of a dibucaine-resistant variant as fluoride-resistant may lead to an erroneous assumption that the latter variant is responsible for the prolonged apnea following succinylcholine. In order to facilitate the correct identification of $E_{1}^{f}$ variant, we recommend the utilization of our modified method for the determination of FN.

\section{CONCLUSION}

Based on the enzyme kinetic studies reported in this article, we recommend the use of $2.5 \times 10^{-4} \mathrm{M} \mathrm{Na}$ fluoride concentration which causes $75 \%$ inhibition of the plasmacholinesterase in $E_{1}^{u} E_{1}^{u}$ at $37 \mathrm{C}$ or body temperature instead of room temperature in order to clearly identify the so-called fluoride-resistant genotypes. The improved selectivity of our modified method for FN allows an accurate identification of the fluoride-resistant genotypes of plasmacholinesterase.

\section{ACKNOWLEDGMENTS}

We are grateful to Ciba Pharmaceuticals of Summit, New Jersey, for the generous supply of dibucaine hydrochloride (nupercaine hydrochloride) and to Ilhan Tuzel, M.D., and Hoffman La Roche, Nutley, New Jersey, for their continued supply of RO2-0683 and for their generous gifts which made these investigations possible.

\section{REFERENCES}

Foldes, F. F., Erdos, E. G., Zsigmond, E. K., and Zwartz, J. A. (1960). Reactivation of neostigmine inhibited human plasma cholinesterase. J. Pharmacol. Exp. Ther. 129:394.

Foldes, F. F., Foldes, V. M., Smith, J. C., and Zsigmond, E. K. (1963). The relation between plasma cholinesterase and prolonged apnea caused by succinylcholine. Anesthesiology 24:208.

Harris, H., and Whittaker, M. (1961). Differential inhibition of human serum cholinesterase with fluoride: Recognition of two new phenotypes. Nature 191:496.

Kothary, S. P., Flynn, K. B., and Zsigmond, E. K. (1979). Fluoride inhibition in patients with atypical dibucaine resistant plasmacholinesterase, Biochem. Genet. 17:1169.

Thompson, J. C., and Whittaker, M. (1966). A study of the pseudocholinesterase in 78 cases of apnea following suxamethonium. Acta Genet. Stat. Med. 16:209.

Whittaker, M. (1967). The pseudocholinesterase variants: A study of fourteen families selected via the fluoride resistant phenotype. Acta Genet. Stat. Med. 17:1.

Whittaker, M. (1968). The pseudocholinesterase variants: Esterase levels and increased resistance to fluoride. Acta Genet. Stat. Med. 18:325.

Whittaker, M., and Vickers, M. D. (1970). Initial experiences with the cholinesterase research unit. Br. J. Anaesthesiol. 42:1016.

Zsigmond, E. K. (1973). Fluoride numbers. Letter to the Editor, Br. J. Anaesth. 45:1053.

Zsigmond, E. K., Flynn, K. B., Matsuki, A., et al. (1973). Atypical plasmacholinesterase as a cause of prolonged apnea 10 years experience with plasma cholinesterase laboratory. Abstracts of Papers, ASA Annual Meeting, October 7-11. 